\title{
Partial reward, the negative contrast effect, and incentive averaging
}

\author{
JAMES H. McHOSE and DOUGLAS P. PETERS \\ Southern Illinois University at Carbondale, Carbondale, Illinois 62901
}

\begin{abstract}
A total of 169 rats, distributed across six experiments, received training in a straight runway with a 5-min intertrial interval. A variety of shifts in reward schedules, to and from partial reward, were employed to assess the effects of partial reward on the successive negative contrast effect. The results were seen as supportive of an incentive averaging approach to partial reinforcement.
\end{abstract}

It is well known that rats originally trained with large reward and subsequently shifted to small reward display a performance level to small reward which is temporarily depressed relative to that of a group trained and maintained on that small reward (cf. Black, 1968; Dunham, 1968). The present paper is concerned with the effects of partial reward training on the depression or negative contrast effect (NCE) associated with reductions in reinforcement.

While the effects of partial reward training on the NCE are of central relevance to theories which purport to identify the mechanisms underlying the behavioral phenomenon (e.g., Capaldi \& Ziff, 1969; Ison, Glass, \& Daly, 1969; Mikulka, Lehr, \& Pavlik, 1967) the present experiments were not directly concerned with an evaluation of these approaches. Rather, the research reported was designed to determine the extent to which the effects of partial reward training on the NCE may be treated within an incentive averaging framework elsewhere shown to apply to the effects of varied and partial reward schedules on the contrast effects associated with differential instrumental conditioning (McHose, 1970; McHose, Maxwell, \& McHewitt, 1971; McHose \& Peters, 1973).

Within an incentive averaging approach, it is first assumed that incentive, $K$, is a negatively accelerated increasing function of the reward amount, A, received on each trial:

$$
\mathrm{K}=\mathrm{M}\left(1-10^{-.10 \mathrm{~A}}\right)
$$

The specific value of the growth parameter, .10, is assigned empirically, based on differential conditioning (cf. McHose \& Peters, 1973) and simple instrumental conditioning experiments (Peters \& McHose, 1974). When $\mathrm{A}$ is varied from trial to trial, as in varied or partial reinforcement schedules, it is assumed that the average incentive value, $\bar{K}$, is a function of the relative frequency, $p$, of the $K$ values established by the different

This research was supported in part by a Special Research Project Grant from the Graduate School, Southern Illinois University. Douglas P. Peters is now at the University of North Dakota. reward amounts contained in the reinforcement schedule:

$$
\overline{\mathrm{K}}=\frac{\mathrm{p}_{1} \mathrm{~K}_{1}, \mathrm{p}_{2} \mathrm{~K}_{2}, \ldots \mathrm{p}_{\mathrm{n}} \mathrm{K}_{\mathrm{n}}}{\mathrm{n}_{\mathrm{k}}}
$$

where $n_{k}$ represents the number of different reward amounts.

Equations 1 and 2 may be applied to the effects of preshift and postshift reinforcement parameters on the NCE with the simple assumption that the NCE results from, and is in proportion to, the decrease in $\overline{\mathrm{K}}\left(\overline{\mathrm{K}}_{\mathrm{DIFF}}\right)$ from the preshift to the postshift period. The observation that the NCE attending a shift to small continuous reward increases with the (constant) amount of preshift reward (DiLollo \& Beez, 1966; Peters \& McHose, 1974) is consistent with this assumption as are the effects of varied preshift reward (Peters \& McHose, 1974) and varied postshift reward (Calef, Hopkins, McHewitt, \& Maxwell, 1973) on the NCE.

\section{EXPERIMENTS I AND II}

The focus of the present paper is on the effects of partial reward training on the NCE. It should be clear that, by Equations 1 and 2, a partial reward schedule would be expected to engender a much lower $\overline{\mathrm{K}}$ value than would comparable continuous reward training, i.e., continuous reward training with the same reward (R)-trial magnitude as employed in the partial schedule. Within the incentive averaging approach, then, the NCE attending a shift to small continuous reward should be greater following large continuous, as compared with large partial, reward training. In this respect, the expectation derived from the averaging approach is comparable to that readily derived from frustration and stimulus specificity theories. Despite the elementary theoretical relevance of such a comparison, there would appear to be little relevant data. Mikulka (1967) found that partial, as compared with continuous large, reward attenuated the NCE observed following a shift to small continuous or partial reward. Ison et al. (1969) found that the NCE obtained following a shift from large to small partial reward approximated that attending a shift 


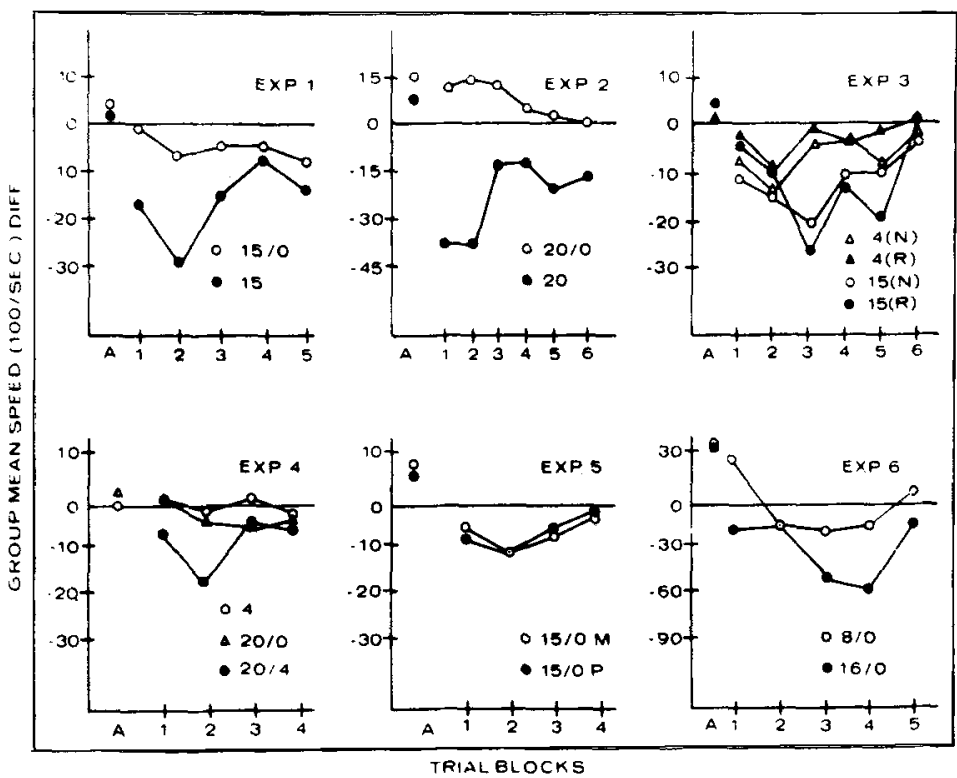

Figure 1. Group mean speed differences as a function of trial blocks for the various conditions of Experiments I-VI. from large to small continuous reward, a result which could be interpreted as contradictory to the Mikulka et al. (1967) finding. Experiments I and II were conducted to further assess the effects of continuous, as compared with partial, reward training on the NCE preparatory to a further evaluation of the incentive averaging approach.

\section{Method}

Subjects. The subjects were 24 (Experiment I) and 30 (Experiment II) experimentally naive, 90-100-day-old, male rats obtained from the Holtzman Company, Madison, Wisconsin.

The apparatus was a straight alley, painted flat black and covered by a translucent Plexiglas ceiling with a $25-\mathrm{cm}-\mathrm{long}$ startbox, an $80-\mathrm{cm}$ run section, and a $25-\mathrm{cm}$ goal section. The inner width and height of each section was $9 \mathrm{~cm}$. A side-exit, solenoid-operated, opaque start door separated the startbox from the running section, and a translucent guillotine-type retrace door separated the run and goal sections. Photocell-clock circuitry provided traversal times over the first three $30-\mathrm{cm}$ segments of the alley. A Pamotor Model 1000A fan mounted on the rear exterior wall of the goalbox extracted air from the apparatus via $30-\mathrm{mm}$ holes in the rear wall.

Procedure. Ten days prior to the first training day (Day 11), subjects were placed on a 23 -h food deprivation regimen which was maintained throughout the experiments. On Days 9 and 10, each subject was handled for approximately $5 \mathrm{~min}$, allowed $2 \mathrm{~min}$ exploration of the alley and start sections of the apparatus, and fed approximately 2045 -mg Noyes pellets, identical to the subsequent reward pellet, in addition to its regular daily feeding.

In Experiment I, eight subjects were randomly assigned to each of three groups labeled according to the number of pellets received following response during the 30 -trial preshift period: Groups $15,15 / 0$, and 1 . Group $15 / 0$ received 15 -pellet reward (R) on two daily trials and was nonrewarded $(N)$ on the remaining trial, according to the following recursive cycle: RNR, RRN, NRR. All groups received one pellet reward on each of the 30 postshift trials. Preshift trials were administered at the rate of three per day. Postshift trials were administered at the rate of 15 trials per day.

The design of Experiment II differed from that of
Experiment I with respect to R-trial magnitude for two groups, the experiment included $20-, 20 / 0$, and 1 -pellet groups $(\mathrm{N}=10)$. In Experiment II, there were 36 preshift trials ( 3 per day) and 36 postshift trials ( 12 per day).

In both experiments, trials were administered to squads composed of six subjects, two from each experimental condition. Each subject in any one squad received its first daily trial before any subject received its second trial, etc. This procedure resulted in an intertrial interval of approximately $5 \mathrm{~min}$.

In both experiments, a running trial was initiated by placing the subject in the startbox. After a 3-sec orientation, the start door was opened. The retrace door was closed following the subject's entrance of the goalbox, and the subject was removed immediately after consumption of the reward or, on $\mathrm{N}$ trials, after $3 \mathrm{sec}$.

\section{Results and Discussion}

In both experiments, as in the subsequent experiments with one exception, the reciprocated total traversal time scores yielded results representative of the scores for the three separate segments of the alley and thus will be the only scores presented.

Group mean speed differences (shifted condition minus unshifted control condition) scores are plotted as a function of blocks of trials in Figure 1. The terminal preshift (Block A) and postshift data of Experiments I and II are presented in the upper left and middle panels of Figure 1, with each trial block containing the data for six trials. As may be seen in Figure 1, the groups which were shifted from continuous large reward to continuous small reward, Groups 15 and 20, displayed postshift speeds which were depressed relative to those of Group 1 (baseline) and to those of their partial reward counterparts, Groups $15 / 0$ and 20/0. Analysis of variance of the data for each of the postshift trial blocks of Experiment I yielded a significant effect of groups at Blocks 1 and 2 (Fs $=13.25$ and 16.70 , $\mathrm{df}=2 / 21$, $\mathrm{p}<.01$ ) and Blocks 3 and 5 (Fs $=4.78$ and 4.82, 
$\mathrm{df}=2 / 21, \quad \mathrm{p}<.05)$. Subsequent pair comparisons (Tukey HSD) indicated that the speeds of Group 15 were reliably $(\alpha=.05)$ depressed relative to those of Group 1 at each trial block and reliably slower than those of Group 15/0 at Blocks 1, 2, and 3. There were no statistically reliable differences between the speeds of Groups 15/0 and 1. In Experiment II, a significant groups effect was obtained at each postshift trial block $(F s=24.71,26.53,4.95,5.12,7.16,4.90 ; \mathrm{df}=2 / 27$, $\mathrm{p}<.05$ for Blocks 1-6, respectively). Subsequent pair comparisons (Tukey HSD) indicated that the speeds of Group 20 differed reliably $(\alpha=.05)$ from those of Groups 20/0 and 1 at each trial block. Group 20/0 ran reliably faster than Group 1 over the first three postshift trial blocks.

The results of both experiments clearly indicate that the NCE attending a shift from large constant reward training are greater than those attending a shift from large partial reward training and, in this respect, are consistent with data obtained by Mikulka et al. (1967). This outcome is consistent with the incentive averaging approach previously outlined, and with mechanism-based interpretations of the NCE, of either the frustration theory (Mikulka et al., 1967) or stimulus specificity (Capaldi \& Ziff, 1969) variety. The current observation that groups shifted from large partial to small continuous reward displayed no convincing NCEs serves as a point of departure for the remaining experiments in this report. From an incentive averaging viewpoint, this finding has the same theoretical import as does the report that small reductions in continuous reward magnitude produce no NCEs (DiLollo \& Beez, 1966; Peters \& McHose, 1974). Indeed, the application of Equations 1 and 2 indicates that the maximum $\overline{\mathrm{K}}$ value obtainable with a $50 \%$ partial reward schedule is approximately equivalent to that engendered by a constant three-pellet reward regimen, with the maximum value obtainable with a $67 \%$ reward schedule approximately equivalent to a five-pellet continuous reward schedule. While it is thus unlikely that shifts from large partial (50\%-67\%) to small continuous reward would yield NCEs, this circumstance does not require the assumption that partial reward uniquely provides for the formation of stimulus-response bonds which attenuate the NCE, an assumption central to frustration based interpretations of partial reward and the NCE (Mikulka et al., 1967).

\section{EXPERIMENTS III AND IV}

Within the averaging framework, the failure to obtain NCEs in the partial reward conditions of Experiments I and II is not a consequence of partial reward train: ing per se but of an insubstantial reduction in $\overline{\mathbf{K}}$ from the preshift to the postshift period. Thus conditions trained on partial reward would be expected to display NCEs if the preshift and postshift reward schedules generated substantially different $\overline{\mathrm{K}}$ s. One way to establish such a situation and concurrently allow for the effective manipulation of preshift reinforcement parameters would appear to be through the use of partial reward schedules in the postshift period. However, relatively little is known about the effects of shifts to partial reward schedules which are of substantially less incentive value than those of the preshift reward schedule. Experiments III and IV were conducted to determine whether shifts to partial reward would yield behavioral effects comparable to those associated with shifts to small continuous reward.

\section{Method}

The subjects were 30 (Experiment III) and 32 (Experiment IV) naive rats of the same description as in Experiments $I$ and II, and the apparatus, as well as the deprivation, habituation, and pretraining procedures were identical to those of Experiments I and II.

In Experiment III, 10 subjects were randomly assigned to each of three groups labeled according to reward conditions over the 30-trial preshift period: Groups 15,4 , and $4 / 0$. Group $4 / 0$ received $50 \%$ reward on a double alternation cycle, with one-half the subjects within this group beginning training with an $\mathrm{N}$ trial, one-half with an $R$ trial. All subjects were administered this schedule during the 36-trial postshift period, half of the subjects within Groups 15 and 4 starting the postshift period with an $R$ trial. Trials were administered at the rate of three per day throughout the experiment.

In Experiment IV, eight subjects were randomly assigned to each of four groups, labeled according to reward conditions during the 36-trial preshift period: Groups $20 / 4,20 / 0,4$, and 4/0. Groups $20 / 4$ and $20 / 0$ received their larger (L) and smaller (S) preshift reward events according to the following recursive cycle: LSL, LLS, SLL. Group $4 / 0$ received $50 \%$ reward on a double alternation cycle, with the group again subdivided according to whether subjects began training with an $N$ or an $R$ trial. All subjects were administered the $4 / 0$ schedule during the 21-trial postshift period, with half of the subjects in Groups $20 / 4,20 / 0$, and 4 beginning the postshift period with an $\mathbf{R}$ trial. Trials were administered at the rate of 3 per day during the preshift period, 12 on the first postshift day, 9 on the last postshift day.

Squad procedures in both experiments were comparable to those detailed for Experiments I and II with the exception that all squads began training on the same calendar day in Experiment III.

\section{Results and Discussion}

Terminal preshift (Block A) and postshift group mean total speeds for Groups 15 and 4 relative to those of the control 4/0 condition are plotted as a function of blocks of six total trials in the upper right panel of Figure 1. At each postshift block, speeds following an $R$ trial are plotted separately from those following an $\mathrm{N}$ trial, with the baseline for each trend line representing the performance level of the $4 / 0$ control condition on trials of the same ordinal position within a block. As may be seen, Group 15 developed a larger depression of postshift speeds relative to Group 4/0, i.e., a larger NCE, that did Group 4. There appeared to be no systematic difference in the extent of the NCE following an R as compared with an $\mathbf{N}$ trial. Analysis of variance of the 
data for each postshift block, including groups and (after) $\mathrm{R}$ vs. (after) $\mathrm{N}$ as factors, yielded significant groups effects at Block $3(\mathrm{~F}=8.13, \mathrm{df}=2 / 27, \mathrm{p}<.01)$ and Block $4(F=4.21, \mathrm{df}=2 / 27, \mathrm{p}<.05)$. Subsequent paired comparisons (Tukey HSD) showed that the speeds of Group 15 differed reliably $(\alpha=.05)$ from those of Groups 4 and $4 / 0$ at each of these blocks. At no trial block did the Groups by $\mathrm{R}$ vs. $\mathrm{N}$ interaction effect approach significance, the analyses thus failing to yield any evidence that the reliable NCE in Group 15 varied with the reward event of the preceding postshift trial.

The terminal preshift and postshift total speeds obtained in Experiment IV are plotted in the lower left panel of Figure 1. Each trial block contains the data for three trials. No systematic effects of the after-R vs. after- $\mathrm{N}$ variable on postshift performance were observed, and the data presented are therefore collapsed across this variable at each trial block. As may be seen in Figure 1, only Group 20/4 displayed a substantial NCE, running slower than the control $4 / 0$ condition over the early portions of the postshift period. Analysis of variance of the data for each postshift trial block yielded a significant groups effect at Block 2 only $(F=4.18$, $\mathrm{df}=3 / 28, \mathrm{p}<.05$ ), with subsequent pair comparisons indicating that Group 20/4 ran reliably $(\alpha=.05)$ slower than the other three groups which, in turn, did not differ from each other. The After-R vs. After- $\mathrm{N}$ by Groups interaction effect did not approach statistical significance at any block of postshift trials.

The collective results of Experiments III and IV suggest that, in several respects, shifts to partial reward schedules produce results analagous to those obtained with shifts to small continuous reward. First, the fact that in Experiment III, Group 15 displayed a substantial NCE while Group 4 did not is consistent with the finding that the NCE attending a shift to small continuous reward increases with preshift reward amount (DiLollo \& Beez, 1966; Peters \& McHose, 1974). Secondly, the occurrence of an NCE in Group 20/4 but not Group 4 (Experiment IV) is comparable to the effect of these schedules on the NCE following a shift to small continuous reward (Peters \& McHose, 1974). Finally, the observation that Group 15 (Experiment III) displayed an NCE while Group 20/0 (Experiment IV) did not, suggests an attenuating effect of partial reward which is analagous to the effects of partial reward observed in Experiments I and II following shifts to small continuous reward. Despite the preceding similarities, it is conceivable that the extent of the NCE on any trial following a shift to partial reward would depend upon the reinforcement event on the preceding postshift trial, thus differentiating partial from continuous postshift reward schedules. In the present data, however, the NCE was not regulated by the reward event on the preceding trial.

\section{EXPERIMENTS V AND VI}

The data of Experiments I-IV are readily interpreted through the application of Equations 1 and 2 and the assumption that the magnitude of the NCE is directly related to the difference between preshift and postshift incentives $\left(\overline{\mathrm{K}}_{\mathrm{DIFF}}\right)$. The failure to obtain NCEs following partial reward training in Experiments I-IV, as well as in previous data (Mikulka et al., 1967) presumably reflects the fact that, in these studies, $\overline{\mathrm{K}}_{\text {DIFF }}$ was minimal. The data of Experiments III and IV further suggest that the use of a postshift partial reward schedule itself has no peculiar effects on the NCE and can thus reasonably be used to maximize $\bar{K}_{\text {DIFF }}$ following partial reward training. Equation 1 implies that there are two different ways in which to produce a substantial $\overrightarrow{\mathrm{K}}_{\text {DIFF }}$ following training on partial reward-reductions in R-trial magnitude and reductions in percentage reinforcement. Experiment $V$ tested the hypothesis that both of these reductions in $\overline{\mathrm{K}}$ would yield NCEs following partial reward training, while Experiment VI varied the amount of R-trial reward reduction between groups.

\section{Method}

The subjects were 32 (Experiment V) and 21 (Experiment VI) experimentally naive rats of the same description as in Experiments I-IV. The apparatus, deprivation, habituation, and prefeeding procedures were as previously described.

In Experiment $\mathrm{V}$, eight subjects were randomly assigned to each of two experimental and two control (C) conditions: Groups 15/0 (M), 15/0 (P), 1/0 (C), and 15/0 (C). Groups 15/0 (M) and $15 / 0$ (P) received identical preshift schedules of $75 \%$ reward, with the daily trial position of $\mathrm{N}$ trials counterbalanced over days. Group $1 / 0$ (C) received the same reinforcement schedule except that R-trial magnitude was one pellet. Group $15 / 0$ (C) received $25 \%$ reward, with the daily trial position of $\mathrm{R}$ trials counterbalanced over days. During the postshift period, Group 15/0 (M) was shifted to the 1/0 (C) condition while Group $15 / 0$ (P) was shifted to the $15 / 0$ (C) condition. The control conditions continued to receive their respective preshift reinforcement schedules. There were 32 preshift and 32 postshift trials, administered at the rate of four per day.

In Experiment VI, seven subjects were assigned to each of three groups, labeled according to their reward schedule during the 40-trial preshift period: Groups $16 / 0,8 / 0$, and 1/0. All subjects received $75 \%$ reward with the daily trial position of $\mathrm{N}$ trials counterbalanced over days. During the 40-trial postshift period, all subjects were shifted to the $1 / 0$ reward schedule. Trials were administered at the rate of four per day throughout the experiment.

Squad procedures were comparable to those previously described. All squads within an experiment began training on the same calendar day.

\section{Results and Discussion}

Group mean total speed difference scores as a function of blocks of eight trials for the conditions of Experiment $\mathrm{V}$ are plotted in the lower middle panel of Figure 1. The speeds for Group 15/0 (M) are expressed as differences from those of its unshifted control condition, Group 1/0 (C), while the speeds for the Group 15/0 (P) are expressed as differences from those of its unshifted control condition, Group 15/0 (C). As may be seen in Figure 1, a reduction in R-trial reward amount produced an NCE, Group $15 / 0(\mathrm{M})$ displaying 
slower postshift speeds than Group 1/0 (C), as did a reduction in percentage reward, Group 15/0 (P) running slower than its control condition. Analysis of variance of the postshift data yielded a significant groups effect at Block $2(\mathrm{~F}=5.66, \mathrm{df}=3 / 16, \mathrm{p}<.01)$. Pair comparisons (Tukey HSD) at this block indicated that both Groups 15/0 (M) and 15/0 (P) differed reliably $(\alpha=.05$ ) from their respective control groups. There were no statistically reliable differences between the absolute postshift performance levels of Groups $1 / 0(\mathrm{C})$ and $15 / 0$ (C) or between those of Groups 15/0 (M) and 15/0 (P).

In Experiment VI, the only response measure in which an NCE was apparent was the third or goal measure. Group mean goal speeds for Groups $16 / 0$ and $8 / 0$ relative to those of the $1 / 0$ control group are plotted as a function of blocks of eight trials in the lower right panel of Figure 1. Inspection of Figure 1 indicates that only Group 16/0 displayed a substantial NCE. Variance analysis of the data at each trial block yielded a significant groups effect at Blocks 3 and $4(\mathrm{Fs}=4.62$, $5.99, \mathrm{df}=2 / 18, \mathrm{p}<.025)$. Pair comparisons showed that, at each of these blocks, Group 16/0 ran reliably $(\alpha=.05)$ slower than Groups $1 / 0$ and $8 / 0$, there being no significant difference between the latter two groups.

In both Experiments V and VI, NCEs were obtained following partial reward training by reducing R-trial magnitudes from the preshift to the postshift period. Moreover, the results of Experiment VI indicate that the NCE produced by a reduction in R-trials magnitude is dependent upon the extent of that reduction. That such reductions produce NCEs would appear to be consistent with data reported by Ison et al. (1969) although the latter findings are difficult to relate to the present results, and to the experimental literature in general, because the NCEs obtained following both partial and continuous reward training appeared to be "permanent" in character, a distinctly atypical result.

In Experiment $V$, a $\overline{\mathrm{K}}_{\mathrm{DIFF}}$ created by a reduction in percentage reinforcement also proved sufficient to yield an NCE following partial reward training.

\section{GENERAL DISCUSSION}

The present results, in conjunction with previous findings, systematically support the application of an incentive averaging framework to describe the effects of various preshift and postshift reward parameters on the NCE. Thus, the averaging approach would appear capable of describing the effects of variations in the conditions of continuous, partial, and varied preshift reward on NCEs attending shifts to small continuous or partial reward, and, perhaps, to varied-magnitude postshift reward schedules (Calef et al., 1973). The extent to which the averaging model suffices to account for the presence or absence of an NCE following a shift in reinforcement conditions may be seen in Table 1, in which the results of 24 different NCE-type comparisons
Table 1

Presence (+) or Absence (-) of an NCE Across Experiments

\begin{tabular}{|c|c|c|c|c|}
\hline \multirow{2}{*}{$\begin{array}{l}\text { Preshift } \\
\text { Schedule }\end{array}$} & \multicolumn{4}{|c|}{ Range of $\overline{\mathrm{K}}_{\text {DIFF }}$} \\
\hline & $26-35$ & $36-45$ & $46-55$ & $56-$ \\
\hline $\begin{array}{l}\text { Partial } \\
\text { Varied } \\
\text { Continuous }\end{array}$ & $\begin{array}{l}+,-,-\mathbf{a},-\mathbf{a} \\
-,-\end{array}$ & $\begin{array}{l}-,+b \\
+,-{ }^{c} \\
-,+d\end{array}$ &,++ & $\begin{array}{l}+,+ \\
+,+,+ \\
+,+,+,+\end{array}$ \\
\hline
\end{tabular}

Note $-\bar{K}_{\text {DIFF }}$ values were derived from Equations 1 and 2 . Entries are from the present data, those of Peters and McHose (1974), and (a) Mikulka et al., 1967; (b) Ison et al., 1969; (c) Davis and North, 1967; (d) Calef et al., 1973.

across experiments are tallied. The data summarized are from the present experiments or previously reported data from this laboratory (Peters \& McHose, 1974), except as noted. As may be seen, the regularity with which NCEs have been observed increases with the amount of incentive reduction from the preshift to the postshift period $\left(\overline{\mathrm{K}}_{\mathrm{DIFF}}\right)$, regardless of the preshift reward schedule. The only entry markedly inconsistent with this summary, an NCE following partial reward training with $26<\mathrm{K}_{\text {DIFF }}<35$ (Peters \& McHose, 1974, Experiment III), would now appear to be simply inconsistent with both previous and subsequent data. The generality of the averaging approach is further enhanced by the observation that it is suitable for describing the effects of variations in continuous, partial, and varied reward schedules on the contrast effects obtained in the differential instrumental conditioning paradigm (McHose, 1970; McHose et al., 1971; McHose \& Peters, 1973).

Despite the generality of the averaging approach to the effects of varied, constant, and partial reward in several contexts, as well as its present simplicity, there are certain empirical and conceptual limitations to the approach as currently developed. Conceptually, the average approach as applied to the NCE assumes a quantity, $\overline{\mathrm{K}}_{\mathrm{DIFF}}$, based upon a difference between an incentive level established by previous training and one appropriate to current reward events. Such an assumption needs little detail to apply to the present two-stage paradigms. However, the application of the averaging notion to multistage paradigms (Capaldi \& Lynch, 1967) will require detailed assumptions regarding the number of reward events over which subjects "average," a matter of interest in several experimental contexts (cf. Baum, 1973).

The chief empirical limitation on the current averaging approach stems from the observation that the partial and varied reward data which support an averaging approach most frequently involve "pseudorandom" reward schedules in which the probability of any specific reward event on one trial is independent of the reward event on the preceding trial. When rats are administered a fixed pattern of rewarded $(\mathrm{R})$ and nonrewarded $(\mathrm{N})$ trials on each of several 
preshift training days, the specific pattern of $R$ and $N$ events influences the NCE despite the fact that, from the present viewpoint, the different patterns yield identical $\widetilde{K} s$ (Capaldi \& Ziff, 1969). An effect of R-N patterns on contrast effects in differential conditioning has also been obtained (McHose \& Blackwell, 1975). The application of averaging theory to the effects of pattern or order of reward events can be accomplished by the ad-hoc assignment of different incentive-determining weights to the successive reward events within a training day. However, such a piecemeal addition to the model would appear unwarranted at this time, particularly in view of the fact that previous data do not determine whether the effects of pattern of reward on the NCE are attributable to the sequence of events within a day or to the order of those events.

As previously mentioned, the present experiments were not designed to provide tests of current competing theories of the NCE, theories which are, in principle, applicable to the effects of various reinforcement parameters on the NCE but which derive their primary descriptive value in other contexts. Stimulus specificity theory as elaborated by Capaldi and his associates is an excellent example of a theory developed to describe extinction data but occasionally applied to the NCE (Capaldi, 1972; Capaldi \& Lynch, 1967; Capaldi \& Ziff, 1969). From the viewpoint of specificity theory, some of the comparisons between groups in the present experiments are exceedingly complex because the reward-produced stimuli to which subjects are exposed and the amount of habit and inhibition acquired to those stimuli are all varied between groups. Consideration of one variable differentiating groups yields a different prediction than consideration of a second variable, etc. In the absence of explicit assumptions concerning the "weights" to be assigned to the various habit and inhibition factors, the present experiments are largely outside the boundary conditions of specificity theory. The present viewpoint is that incentive averaging and specificity approaches, aside from the language employed, are not necessarily contradictory. Rather, the averaging principle may simply be seen as one ultimately derivable from specificity theory when the aforementioned explicit assumptions are made. Alternatively, the averaging principle might be incurporated in the set of assumptions comprising simulus specificity theory. Indeed, Capaldis (1974) recent suggested concept of "reinforcement level" would appear to be quite similar to an incentive averaging notion.

\section{REFERENCES}

Baum, W. M. Correlation-based law of effect. Journal of the Experimental Analy sis of behavior, 1973, 20, 137-153.

Black, R. W. Shifts in magnitude of reward and contrast effects in instrumental and selective learning: A reinterpretation. Psychological Review, 1968, 75, 114-126.

Calef, R. C., Hopkins, D. C.. McHewitt, E. R., \& Maxwell, F. R Performance to varied reward following continuous reward training in the runway. Bulletin of the Psychonomic Society, $1973,2,103-104$.

Capaldi, E. J. Successive negative contrast: Intertrial interval, type of shift, and four sources of generalization decrement. Journal of Experimental Psychology, 1972, 96, 433-438

Capaldi, E. J. Partial reward either following or preceding consistent reward: A case of reinforcement level. Journal of Experimental Psychology, 1974, 102,954-962.

Capaldi, E. J., \& Lynch, D. Repeated shifts in reward magnitude Evidence in favor of an associational and absolute (noncontextual) interpretation. Journal of Comparative and Phy siological Psy chology, 1967, 75, 226-235.

Capaldi, E. J., \& Ziff, D. R. Schedule of partial reward and the negative contrast effect. Journal of Comparative and Physiological Psychology, 1969,68,593-596.

Davis. S, F., \& North, A. J. The effect of varied reinforcement training on behavior following incentive reduction. Psychonomic Science, 1967, 9, 395-396.

DiLollo, $V$. \& Bee $2, V$. Negative contrast effects as a function of magnitude of reward decrement. Psy chonomic Science, 1966. $5,99-100$.

Dunham, P. J. Contrasted conditions of reinforcement: A selective critique. Psychological Bulletin, 1968, 69, 295-315,

Ison, J. R., Glass, D. H. \& Daly, H. B. Reward magnitude changes following differential conditioning and partial reinforcement. Joumal of Experimental Psychology, 1969 , 81. 81-88

McHose, J. H. Relative reinforcement effects: $S_{1} / S_{2}$ and $S_{1} / S_{1}$ paradigms in instrumental conditioning. Psychological Review, $1970,77,135-146$.

McHose, J. H.. \& Blackwell, D. R. Performance in differential instrumental conditioning as a function of the pattern of $\mathrm{S}+$ reward. Animal Learning and Behavior, 1975, 3, 63-66.

McHose, J. H., Maxwell, F. R., \& McHewitt, E. R. Effects of nonreward in $S+$ and $S-$ on performance in differential conditioning. Joumal of Experimental Psy chology, 1971, 88, 282-284

McHose, J. H., \& Peters, D. P. Differential instrumental conditioning as a function of the percentage and amount of St reward. Joumal of Experimental Psy chology, 1973, 100 , $413-415$

Mikulka, P. J., Lehr, R., \& Pavlik, W. B. Ef fects of reinforcement schedules on reward shifts. Joumal of Experimental Psy chology, 1967, 74, 57-61.

Peters, D. P., \& McHose, J. H. Ef fects of varied preshift reward magnitude on the successive negative contrast effect in rats. Journal of Comparative and Physiological Psychology, 1974 , 86, 85-95.

(Received for publication September 24, 1974; accepted February 13,1975.) 